\title{
Avaliação de Protocolo de Prevenção e Tratamento de Úlceras de Pressão*
}

\section{Evaluation of a Prevention Protocol of Pressure Ulcers}

\author{
Marisol Louro' ${ }^{1}$, Margareth Ferreira ${ }^{1}$, Pedro Póvoa ${ }^{2}$
}

\section{RESUMO}

JUSTIFICATIVA E OBJETIVOS: As úlceras de pressão (UP) constituem um importante problema de saúde, em particular nas unidades de terapia intensiva (UTI). O objetivo deste estudo foi identificar o número, grau e escore total das UP na admissão, durante a internação e na alta. Determinar quais os fatores que influenciaram o seu aparecimento e desenvolvimento, assim como verificar a sua incidência e prevalência.

MÉTODO: Todos os pacientes internados na UTI por período superior que 24 horas foram, prospectivamente, incluídos e acompanhados durante um ano. Foram excluídos 70 pacientes por dados insuficientes. A todos foi aplicado o mesmo protocolo de prevenção e de tratamento (placas hidrocolóides, hidrogel, se necrose presente e alginato de cálcio, se UP com sangramento).

RESULTADOS: Foram estudados 155 pacientes. Dezoito apresentavam UP na admissão e 40 a desenvolveram durante a internação, totalizando 125 UP. A prevalência foi de $37,41 \%$ e a incidência de $25,8 \%$. O aparecimento de novas UP ocorreu, em média, no $7^{\circ}$ dia de internação. Dos pacientes internados $79 \%$ mantiveram-se estáveis e/ou melhorados. O SAPS 2 dos

\footnotetext{
1. Enfermeira Graduada do Hospital Garcia de Orta, Almada

2. Assistente Hospitalar Graduado em Medicina Interna, Hospital São Francisco Xavier - Lisboa

*Recebido do Hospital Garcia de Orta, Almada, Portugal

Apresentado em 11 de abril de 2007

Aceito para publicação em 04 de julho de 2007

Endereço para correspondência:

Margareth Ferreira

Praceta João de Barros, $\mathrm{n}^{\circ} 5-5 \mathrm{dt}^{\circ}$

Torre da Marinha

2840-410 - SEIXAL

Portugal

Telemovel - +351961406432

E-mail: maggie.f@sapo.pt
}

(C)Associação de Medicina Intensiva Brasileira, 2007 pacientes admitidos com UP foi superior aos dos pacientes sem UP, $54 \pm 8,7$ e $44 \pm 17$, respectivamente ( $p$ $=0,015)$. Na alta o número de UP foi significativamente maior nos pacientes classificados como de alto risco avaliados pela escala de Norton adaptada $(p=0,039)$. Os pacientes que evoluíram para óbito apresentaram mais UP que os demais $(p<0,001)$. Foi constatado que quanto maior o tempo de internação maior a prevalência de UP à data da alta $(p<0,001)$.

CONCLUSÕES: Na população estudada as UP apresentaram prevalência de $37,41 \%$ e incidência de $25,8 \%$. A aplicação do protocolo de prevenção de UP foi eficaz em $79 \%$ dos pacientes, sendo que as UP apareceram com maior freqüência nos pacientes mais graves.

Unitermos: escore total de úlcera, grau de risco, grau total de úlcera, número total de UP, UCI, úlcera de pressão

\section{SUMMARY}

BACKGROUND AND OBJECTIVES: Pressure ulcers (PU) constitute an important health problem in particular in the intensive care unit (ICU). The objective of the study was to identify the number, degree and total score of PU on admission, ICU stay and discharge as well as to recognize factors influencing the appearance or development of PU and to identify the number of healed PU, thus so the incidence and prevalence.

METHODS: All patients admitted $>24$ hrs were prospectively included during one year. Seventy patients were excluded for insufficient data. The prevention protocol (Norton scale; positioning according the risk grade) and therapeutic protocol (hydrocolloid dressings; hydrogel dressings if tissue necrosis and/or devitalized and alginate dressings if ulcer bleeds) was applied to all patients.

RESULTS: One hundred and fifty five patients were studied. Eighteen patients were admitted already with PU. During ICU stay, 40 patients developed a total of 125 
PU. The prevalence of PU was $37.41 \%$ and incidence was $25.8 \%$. The development of new PU occurred on average by the 7th day. Patients with PU presented 2.6 $\mathrm{PU}$ on the average. Seventy nine percent of the patients admitted in the ICU remained stable or improved. Patients admitted with PU had a SAPS 2 significantly higher than those without, $54 \pm 8.7$ and $44 \pm 17$, respectively $(p=0.015)$. At the day of discharge, patients classified as high risk had significantly more PU $(p=$ 0.039). Non-survivors had significantly more PU than survivors $(p<0.001)$. Patients with longer ICU stay had more PU $(p<0.001)$

CONCLUSIONS: In our patient population we found $37.41 \%$ prevalence and $25.8 \%$ incidence of PU. The present prevention protocol of PU was effective in $79 \%$ of the patients; severely ill patients developed PU more frequently.

Key Words: ICU, grade, pressure ulcer, total grade of $\mathrm{PU}$, total score of PU, total number of PU

\section{INTRODUÇÃO}

As úlceras de pressão (UP) são definidas como "Uma área de lesão localizada da pele e dos tecidos subjacentes, causadas por pressão, tensão tangencial, fricção e/ ou uma combinação destes fatores" ". Elas constituem um importante problema com que os profissionais da saúde freqüentemente se deparam. Custam e afetam milhões de pacientes, nos lares, nos centros de saúde, nas instituições hospitalares e, em particular, nas unidades de terapia intensiva (UTI). De acordo com os dados epidemiológicos publicados, a taxa de incidência e prevalência destas lesões é maior nas UTI ${ }^{2,3}$.

A Organização Mundial de Saúde (OMS) utiliza a incidência e a prevalência das UP como um dos indicadores para determinar a qualidade dos cuidados prestados ${ }^{4}$.

Cerca de 95\% das UP são evitáveis ${ }^{5}$, pelo que se torna imprescindível utilizar todos os meios disponíveis para realizar uma eficaz prevenção e tratamento das UP já estabelecidas. Estas preocupações motivaram a criação de um grupo de enfermeiras que durante o ano de 2000 elaborou um protocolo de prevenção e tratamento de UP. Como em qualquer trabalho surgiu a necessidade de proceder a sua avaliação.

O objetivo do presente estudo foi determinar a taxa de incidência e prevalência das úlceras de pressão na UTI; identificar o número, grau e escore total das úlceras de pressão na admissão, internação e alta; determinar quais os fatores influenciaram o seu aparecimento e desenvolvimento e identificar o número de UP cicatrizadas.

\section{MÉTODO}

Este estudo foi realizado em UTI geral (médicos, cirúrgicos e trauma) de nível III com capacidade para oito leitos.

Trata-se de um estudo descritivo, prospectivo no qual foram incluídos todos os pacientes internados em 2002, com internação superior a 24 horas. Para cada paciente foram registrados os seguintes dados: sexo, idade, APACHE II, SAPS 2, dias de internação, diagnóstico, aplicação do protocolo de prevenção, dia de aparecimento grau e escore da UP.

Dos 225 pacientes internados durante o estudo, selecionou-se 155 pacientes.

Para calcular a prevalência (mediu-se a proporção de pessoas que inicialmente não apresentaram UP, mas as desenvolveram em determinado período) e a incidência (mediu-se a proporção de pessoas que desenvolveram UP em determinado momento) das UP seguiram-se as diretrizes do Grupo Nacional para o Estudo e Assessoria em Úlceras de Pressão - Espanha (GNEAUPP) ${ }^{7}$.

Todos os leitos eram dotados de uma superfície estática, isto é de um colchão de redução da pressão; durante a realização do estudo a unidade não possuía colchões de pressão alterna ${ }^{6}$.

Sempre que ocorria alteração na integridade da pele, essa era descrita com detalhe na folha de registro criada para tal efeito. Neste registro anotavam-se todas as características da lesão: grau da úlcera, localização, escore, tratamento aplicado e data prevista do próximo tratamento.

Para classificar as UP quanto ao grau de profundidade, foi adotada a classificação publicada pela European Pressure Ulcer Advisory Panel (EPUAP) e GNEAUPP ${ }^{1,9}$ que define:

Grau I - eritema não branqueável em pele intacta, a lesão precursora da pele. Em pacientes de pele escura, o calor, o edema, o endurecimento ou a dureza também podem ser indicadores;

Grau II - perda parcial da pele, que envolve a epiderme a derme ou ambas (abrasão/flictena);

Grau III - perda de espessura total da pele, podendo incluir lesões ou mesmo necrose do tecido subcutâneo, com extensão até a fáscia subjacente, mas não através dessa;

Grau IV - destruição extensa, necrose dos tecidos ou 
lesão muscular e/ou exposição óssea ou das estruturas de apoio (neste grau como no III, podem apresentar lesões com cavernas, túneis ou trajetos sinuosos).

A avaliação da evolução da cicatrização das UP foi realizada através da escala de cicatrização publicada pela GNEAUPP. Esse instrumento é constituído por três dimensões: tipo de tecido, quantidade de exsudado e área da úlcera ${ }^{10}$.

Para efeitos de análise os pacientes foram classificados em:

Pacientes melhorados - os que apresentaram UP durante a sua internação na UTI (adquirida na UTI ou não) e em que o escore total das UP na alta foi inferior ao escore total das UP na internação;

Pacientes estáveis - os admitidos na UTI sem UP e que durante a internação não a desenvolveram e todos os que na admissão na UTI apresentaram UP e que não se observou alteração do grau ou escore durante a internação.

O dia da variação da UP era o $1^{\circ}$ dia da internação, em que se verificou o aparecimento de uma ou mais UP ou alteração do grau ou escore da UP já existente.

O protocolo de prevenção consistiu em avaliar os pacientes através da escala de Norton nas primeiras 24 horas de internação, o que permitiu uma classificação dos pacientes por graus de risco. Definiram-se 4 graus: a) alto risco, b) médio risco, c) baixo risco e d) sem risco.

A periodicidade de reavaliação dos pacientes ${ }^{8}$ dependia do grau de risco que apresentavam (alto $-5 / 5$ dias; médio - 4/4 dias; baixo e sem risco - 3/3 dias). Os cuidados de higiene e conforto foram realizados sempre que necessário; o posicionamento e a aplicação de creme hidratante a cada duas horas aos pacientes de alto risco, a cada três horas aos de médio risco e uma vez por turno aos de baixo risco.

Em relação ao protocolo de prevenção, foi registrada a freqüência em que o protocolo foi aplicado e que deveria ter sido aplicado e foi elaborada a seguinte escala: raramente: $0 \%$ a $25 \%$; algumas vezes: $26 \%$ a $50 \%$; freqüentemente: $51 \%$ a $75 \%$; sempre: $76 \%$ a $100 \%$.

O protocolo terapêutico consistiu em:

1) UP de grau I e II, aplicação de placas hidrocolóides; 2) UP de graus III e IV aplicação de placas hidrocolóides e hidrogel, se tecido necrosado ou/e desvitalizado e alginato de cálcio, se úlcera com sangramento.

\section{Análise Estatística}

Os resultados estão apresentados como média \pm desvio-padrão a não ser quando especificado de outra for- ma. As comparações entre grupos foram feitas recorrendo aos testes $t$ de Student não pareado, One-Way, ANOVA, Mann-Whitney $\mathrm{U}$ ou Kruskal-Wallis $\mathrm{H}$ para as variáveis contínuas e de acordo com a distribuição. Comparações múltiplas post-hoc foram realizadas recorrendo ao teste de Bonferroni.

Comparações entre as variáveis categóricas foram realizadas com os testes Qui-quadrado e Exato de Fisher. As correlações entre 2 variáveis, numéricas ou categóricas foram realizadas calculando o coeficiente de correlação (r) ou a correlação de Spearman (rs). Considerou-se significativo quando $p<0,05$.

\section{RESULTADOS}

Foram estudados 155 pacientes com média de idade de 63 anos, sendo $63 \%$ do sexo masculino e APACHE II $20 \pm 8$. Os dados estão apresentados na tabela 1 .

\begin{tabular}{lc} 
Tabela 1 - Caracterização dos Pacientes Estudados $(\mathrm{n}=155)$ \\
\hline \multicolumn{2}{l}{ Variáveis } \\
\hline Idade (anos) & 63 \\
Sexo (\%) & \\
$\quad$ Masculino & 63 \\
$\quad$ Feminino & 37 \\
APACHE II * & $20 \pm 8$ \\
Clínicos & $70 \%$ \\
Cirúrgicos & $30 \%$ \\
Mortalidade & $36 \%$ \\
\hline
\end{tabular}

* Valor expresso em Média \pm DP

Dos 155 pacientes, 18 apresentavam UP na admissão. Nesses o número de UP por paciente foi de 1,27, com grau e escore médio de 1,7 e 8,2, respectivamente. Durante a internação, 40 pacientes desenvolveram UP, num total de 125 UP. A prevalência foi de $37,41 \%$ e a incidência de 25,80\%.

O aparecimento de novas UP ocorreu, em média, no $7^{\circ}$ dia de internação e o seu número variou em média para mais 1,4 úlceras. Em média o grau e o escore das UP aumentaram para 2,9 e 12,1, respectivamente. Os pacientes com UP apresentaram em média 2,6. A maioria dos pacientes não teve dias de imobilidade, contudo os que apresentavam UP, foram os que tiveram mais dias de imobilidade. Nos pacientes sem risco, nenhum desenvolveu UP. Só ocorreram UP nos pacientes com algum grau de risco e quanto mais elevado o risco, maior o número de pacientes com UP. Foram cicatrizadas 20 UP. Alguns apresentaram outras UP no momento da alta, num grau total médio de 2,2 e escore total médio de 9,2. Dos pacientes internados $79 \%$ mantiveram-se estáveis 
e/ou melhorados. O SAPS 2 dos pacientes admitidos com UP versus sem UP foi superior, $54 \pm 8,7$ e $44 \pm 17$, respectivamente $(p=0,015)$. O grau e escore total das UP foram significativamente inferiores nos pacientes em que se aplicou o protocolo de prevenção $(p=0,008$ e $p=0,011$ ) (Tabela 2). Na alta o número de pacientes com UP foi significativamente maior nos classificados como de alto risco $(p=0,039)$ (Tabela 3$)$. Os falecidos apresentaram número de significativamente maior de UP aos sobreviventes na data da alta da UTI $(p<0,001)$ (Tabela 4). Quanto maior o tempo de internação, maior o número de UP na alta $(p<0,001)$ (Tabela 5$)$.

Tabela 2 - Aplicação do Protocolo de Prevenção

\begin{tabular}{lccc}
\hline & Habitualmente & Ocasionalmente & Valor de $\mathrm{p}$ \\
\hline Escore & $3,6 \pm 7,6$ & $10,4 \pm 17,6$ & 0,008 \\
Grau & $0,8 \pm 1,7$ & $2,5 \pm 4,2$ & 0,011 \\
\hline
\end{tabular}

Tabela 3 - Número de Pacientes com Úlcera de Pressão na Alta

\begin{tabular}{lcc}
\hline Graus de Risco & Sem UP & Com UP \\
\hline Alto & 28 & 35 \\
Médio & 35 & 13 \\
Baixo & 27 & 6 \\
Sem risco & 11 & 0 \\
\hline
\end{tabular}

$p=0,039$

Tabela 4 - Prevalência das Úlceras de Pressão nos Sobreviventes e Óbitos

\begin{tabular}{lcc}
\hline & Sem UP & Com UP \\
\hline Óbito & 24 & 31 \\
Sobrevivente & 77 & 23 \\
\hline
\end{tabular}

$(p<0,001)$

Tabela 5 - Relação entre Tempo de Internação e Desenvolvimento de Úlceras de Pressão

\begin{tabular}{lcc}
\hline Dias & Com UP & Sem UP \\
\hline 0 a 15 & 26 & 81 \\
16 a 30 & 17 & 12 \\
31 a 45 & 11 & 3 \\
$\geq 46$ & 4 & 1 \\
\hline
\end{tabular}

$(p<0,001)$

\section{DISCUSSÃO}

Após o estudo verificou-se que o aparecimento de novas UP ocorreu, em média, no $7^{\circ}$ dia de internação, assim como o seu aumento nas internações prolongadas, o que corrobora com outros estudos ${ }^{11,13-15}$.

Pode-se inferir que a prevalência e a incidência obtida está de acordo com outros estudos ${ }^{6,11,12,15}$ (entre 14\% e $41 \%$; e, $1 \%$ e $56 \%$, respectivamente).

O grau de gravidade das UP aumenta em média 2,9, o que está de acordo com o estudo de Bours e col., que refere que os pacientes internados em UTI que apresentam UP de grau I rapidamente se podem deteriorar e passar para grau II ou superior ${ }^{12}$.

A imobilidade é um dos fatores assinalados por muitos autores como determinante para o desenvolvimento de UP, apesar do presente estudo não ter encontrado diferença estatisticamente significativa. Contudo, verificou-se que os pacientes que apresentavam UP eram os que tinham mais dias de imobilidade.

Embora as escalas de avaliação de risco, sejam limitadas no método de construção e validade, podem fornecer uma estrutura e sugestões apropriadas para a avaliação. O fato de não existir uma escala específica para UTI, levou a Agency for Health Care Policy and Research recomendar a utilização da escala de Norton ou de Braden ${ }^{2}$. Neste estudo utilizou-se a escala de Norton e verificou-se que os pacientes sem risco não desenvolveram UP e quanto mais elevado foi o risco, maior era o número de pacientes com UP.

Os pacientes admitidos com UP apresentaram índices de gravidade mais elevados que os admitidos sem UP. Os estudos ${ }^{11,12}$ afirmaram que os índices de gravidade (APACHE II e SAPS 2) são importantes indicadores para a identificação do risco de desenvolvimento de UP. Verificou-se que nos pacientes em que ocorreu o cumprimento na totalidade do protocolo de prevenção, o escore e grau total das UP foi significativamente inferior em relação aos outros. Vários estudos ${ }^{11,12,15}$ descreveram que a prevenção é tão importante como a identificação do risco, razão pela qual devem ser criados escalas e protocolos de prevenção adequada para cada grau de risco, assim como promover a sua correta implementação.

\section{CONCLUSÃO}

A aplicação do protocolo de prevenção foi eficaz em $79 \%$ dos pacientes. O aparecimento das UP ocorreu freqüentemente nos pacientes mais graves e apareceram, em média, no $7^{\circ}$ dia de internação.

Muito tem sido discutida acerca das medidas a serem adotadas na sua prevenção ${ }^{15,16}$. Essa deve ser a prioridade máxima das instituições. Estas medidas passam por equipar as unidades hospitalares com material de alívio de zonas de pressão, monitorizar o grau de risco, incidência e prevalência. Elas devem ser o primeiro passo para a implementação de protocolos de prevenção e para a sensibilização das equipes para a problemática das UP. 


\section{AGRADECIMENTOS:}

A toda equipe de enfermagem da UTI do Hospital Garcia de Orta (Almada, Portugal), pela colaboração no preenchimento e recolhimento dos dados.

\section{REFERÊNCIAS}

01. Diretrizes para a Prevenção de Úlceras de Pressão - European Pressure Ulcer Advisory Panel. Disponível em: http://www.epuap.org. Consultada em 23 de Agosto de 2003.

02. Morison M - Epidemiologia. Prevenção e Tratamento de Úlceras de Pressão. 1ª Ed, Loures: Lusociência, 2001;286.

03. Dados Epidemiológicos em Úlceras por Presión. Disponível em: http:// www.ulceras.net/epidemiologiaenupp.htm. Consultada em 23 de Agosto de 2003.

04. Estudio sobre Úlceras por Presión en un Centro Socio Sanitario. Disponível em: http://www1.dragonet.es/users/d1346/nafres.htm. Consultada em 16 de Dezembro de 2003.

05. Ribeiro F - Feridas e Úlceras Cutâneas. $1^{\mathrm{a}}$ Ed, Coimbra: Formasau Formação e Saúde Lda, 1999;210.

06. Furtado K - Úlceras de Pressão. Prevenção e Tratamento de Úlceras. Formasau. $1^{\text {a }}$ Ed, Coimbra: Formação e Saúde Lda, 2001;9-25

07. Epidemiologia de las Úlceras por Presión - Grupo Nacional para o Estudo e Assessoria em Úlceras de Pressão. Disponível em http///www. gneaupp.org. Consultada em 8 de Setembro de 2003.

08. Narvaez P - Úlceras por Presión - Evaluación de un Protocolo, Rol de Enfermería 1997; 225:73-78.

09. Classificacion de las Úlceras por Presión - Grupo Nacional Para o Estudo e Assessoria em Úlceras de Pressão. Disponível em http///www. gneaupp.org. Consultada em 10 de Agosto de 2003.

10. Monitorización Evolución de las Úlceras por Presión - Grupo Nacional Para o Estudo e Assessoria em Úlceras de Pressão. Disponível em http///www.gneaupp.org. Consultada em 2 de Setembro de 2003.

11. Keller B, Wille J, van Ramshorst B et al - Pressure ulcers in intensive care patients: a review of risks and prevention. Intensive Care Med, 2002;28:1379-1388.

12. Bours GJ, De Laat E, Halfens RJ et al - Prevalence, risk factors and prevention of pressure ulcers in Dutch intensive care units. Results of a cross-sectional survey. Intensive Care Med 2001;27:1599-1605.

13. Eachempati SR, Hydo LJ, Barie PS - Factors influencing the development of decubitus ulcers in critically ill surgical patients. Crit Care Med, 2001;29:1678-1682.

14. Gonzalez-Ruiz JM, Gonzalez-Carrero AA, Heredero-Blázquez MT et al - Factores de riesgo de las úlceras por presión en pacientes críticos. Enfermería Clinica, 2001;5:184-190.

15. Rogenski N, Santos V - Estudo sobre a incidência de úlceras por pressão em um hospital universitário. Rev Latino-Am Enfermagem 2005;13;474480.

16. Ulceras de pressão. Disponível em: http:// www.manualmerck.net. Consultada em 14 de Fevereiro de 2006. 